\title{
UNITS OF GROUP RINGS OF GROUPS OF ORDER 16
}

\author{
by E. JESPERS and M. M. PARMENTER $\dagger$
}

(Received 27 May, 1992)

Let $G$ be a finite group, $\mathscr{U}(\mathbf{Z} G)$ the group of units of the integral group ring $\mathbf{Z} G$ and $U_{1}(\mathbf{Z G})$ the subgroup of units of augmentation 1 . In this paper, we are primarily concerned with the problem of describing constructively $U(\mathbf{Z} G)$ for particular groups $G$. This has been done for a small number of groups (see [11] for an excellent survey), and most recently Jespers and Leal [3] described $\mathcal{U}(\mathbf{Z G )}$ for several 2-groups. While the situation is clear for all groups of order less than 16, not all groups of order 16 were discussed in their paper. Our main aim is to complete the description of $U(\mathbf{Z G})$ for all groups of order 16. Since the structure of the unit group of abelian groups is very well known (see for example [10]), we are only interested in the non-abelian case.

Note that there are 7 indecomposable non-abelian groups of order 16 :

$$
\begin{aligned}
P & =\left\langle a, b \mid a^{4}=1=b^{4}, b a=a^{3} b\right\rangle, \\
Q_{16} & =\left\langle a, b \mid a^{8}=1, a^{4}=b^{2}, b a=a^{7} b\right\rangle, \\
D & =\left\langle a, b, c \mid a^{2}=b^{2}=c^{4}=1, a c=c a, b c=c b, b a=c^{2} a b\right\rangle, \\
D_{16}^{+} & =\left\langle a, b \mid a^{8}=b^{2}=1, b a=a^{5} b\right\rangle, \\
D_{16}^{-} & =\left\langle a, b \mid a^{8}=b^{2}=1, b a=a^{3} b\right\rangle, \\
D_{16} & =\left\langle a, b \mid a^{8}=b^{2}=1, b a=a^{7} b\right\rangle, \\
H & =\left\langle a, b \mid a^{4}=b^{4}=(a b)^{2}=\left(a^{2}, b\right)=1\right\rangle .
\end{aligned}
$$

There are also 2 decomposable non-abelian groups of order 16:

$$
Q \times C_{2} \text { and } D_{8} \times C_{2} \text {, }
$$

where $Q$ is the quaternion group, $C_{2}$ the cyclic group of order 2 and

$$
D_{8}=\left\langle a, b \mid a^{4}=b^{2}=1, b a=a^{3} b\right\rangle,
$$

the dihedral group of order 8 .

Now, a description of $\mathcal{U}(\mathbf{Z} G)$ has been given for $G=P, D, D_{16}^{+}$and $H$ in [3]. It is well known that $\mathbf{Z}\left(Q \times C_{2}\right)$ has only trivial units. So in this paper we will deal with the groups $G=D_{16}^{-}, D_{16}, Q_{16}$ and $D_{8} \times C_{2}$.

Secondly there is the question of whether the bicyclic and Bass cyclic units generate a subgroup of finite index in $U(\mathbf{Z G})$. Recall that a bicyclic unit in $\mathbf{Z} G$ is a unit of the form $1+(1-x) y\left(1+x+\ldots+x^{n-1}\right)$, where $x, y \in G$ and $n$ is the order of $x$. For the definition of Bass cyclic unit, the reader is referred to [9]. For finite nilpotent groups $G$, Ritter and Sehgal [9] showed that under some restrictions the Bass cyclic and the bicyclic units generate a subgroup of finite index in $\mathcal{U}(\mathbf{Z G})$. The restrictions are on the 2-Sylow subgroups, and the situation for 2-groups is still unclear.

In [1] it is proved that the Bass cyclic units generate a subgroup of finite index in $\mathcal{U}(\mathbf{Z} G)$ for all finite abelian groups $G$. Further, it was shown in [8] that the Bass cyclic

† The authors are supported in part by NSERC-grants OGP0036631 and A8775.

Glasgow Math. J. 35 (1993) 367-379. 
and the bicyclic units generate a subgroup of finite index in $U\left(Z D_{2^{\prime \prime}}\right)$ for all dihedral groups $D_{2^{n}}$ of order $2^{n}$. So, again, the first difficulties arise with the non-abelian groups of order 16 (different from $D_{16}$ ). We will show that $D_{16}$ is the only indecomposable non-abelian group of order 16 for which the Bass cyclic and bicyclic units generate a subgroup of finite index in the unit group. The groups $P$ and $Q_{16}$ were dealt with in [9], and $D$ and $D_{16}^{+}$were treated in [4]. So in this paper we complete the story by handling the groups $D_{16}^{-}$and $H$.

Since $Q \times C_{2}$ has only trivial units the above problem is trivial for this group and the only group left is $D_{8} \times C_{2}$. It seems that this should be an easy group to deal with, especially as we obtain a nice description of the full unit group. However we are unable to answer the question concerning the Bass cyclic and bicyclic units for this group.

Throughout we follow the notation of $[10]$.

Crucial to our work is the following result of Jespers and Leal about $\mathcal{U}\left(\mathbf{Z} D_{8}\right)$.

THEOREM $1([3])$. In $U_{1}\left(\mathbf{Z} D_{8}\right)$, $D_{8}$ has a torsion-free normal complement

$$
W=\left\{u=1+\alpha\left(1-a^{2}\right) \mid \text { u a unit, } \alpha \in \Delta_{\mathbf{z}}\left(D_{8}\right)\right\} .
$$

Further, $W$ is a free group of rank 3 generated by any 3 of the 4 distinct (up to inverses) bicyclic units of $\mathbf{Z} D_{8}$.

The proof given in [3] relies on a method to concretely describe the Wedderburn decomposition of the rational group algebra for a class of 2-groups. For the dihedral group of order 8 , another more direct proof of the theorem was given in [6].

1. Description of $\mathcal{U}\left(\mathbf{Z} D_{16}^{-}\right)$. Here we will describe $U(\mathbf{Z} G)$, where

$$
G=D_{16}^{-}=\left\langle a, b \mid a^{8}=b^{2}=1, b a=a^{3} b\right\rangle .
$$

First we examine closely the Wedderburn decomposition of the rational group algebra $\mathbf{Q} G$. We note that $\mathbf{Q} G\left(\frac{1}{2}\left(1-a^{4}\right)\right)$ is 8 -dimensional over $\mathbf{Q}$ and $Z\left(\mathbf{Q} G\left(\frac{1}{2}\left(1-a^{4}\right)\right)\right)$ is 2-dimensional over $\mathbf{Q}$. Observe that $\left(a+a^{3}\right) \frac{1}{2}\left(1-a^{4}\right)$ is in $Z\left(\mathbf{Q} G\left(\frac{1}{2}\left(1-a^{4}\right)\right)\right)$ and $\left[\left(a+a^{3}\right) \frac{1}{2}\left(1-a^{4}\right)\right]^{2}=-2\left(\frac{1}{2}\left(1-a^{4}\right)\right)$. Hence $Z\left(\mathbf{Q} G\left(\frac{1}{2}\left(1-a^{4}\right)\right)\right)$ is the field

$$
\mathbf{Q}\left[\left(a+a^{3}\right) \frac{1}{2}\left(1-a^{4}\right)\right] \cong \mathbf{Q}[\sqrt{-2}] .
$$

Next note that the following 4 elements of $\mathbf{Q} G\left(\frac{1}{2}\left(1-a^{4}\right)\right)$ are linearly independent over $\mathbf{Q}\left[\left(a+a^{3}\right) \frac{1}{2}\left(1-a^{4}\right)\right]$ and, in fact, form an elementary matrix basis:

$$
\begin{array}{ll}
e_{11}=\left(\frac{1+b}{2}\right)\left(\frac{1-a^{4}}{2}\right), & e_{12}=-a^{2}\left(\frac{1-b}{2}\right)\left(\frac{1-a^{4}}{2}\right), \\
e_{21}=a^{2}\left(\frac{1+b}{2}\right)\left(\frac{1-a^{4}}{2}\right), & e_{22}=\left(\frac{1-b}{2}\right)\left(\frac{1-a^{4}}{2}\right) .
\end{array}
$$

Hence $\mathbf{Q} G\left(\frac{1}{2}\left(1-a^{4}\right)\right) \cong M_{2,2}(\mathbf{Q}[\sqrt{-2}])$ and the above remarks indicate exactly how this 
isomorphism works. Specifically, we set $X=\left(a+a^{3}\right) \frac{1}{2}\left(1-a^{4}\right)$, and obtain

$$
\begin{aligned}
a\left(\frac{1-a^{4}}{2}\right) & =\frac{X}{2}\left(e_{11}+e_{22}+e_{12}-e_{21}\right), \\
b\left(\frac{1-a^{4}}{2}\right) & =e_{11}-e_{22}, \\
a^{2}\left(\frac{1-a^{4}}{2}\right) & =e_{21}-e_{12}, \\
a^{3}\left(\frac{1-a^{4}}{2}\right) & =\frac{X}{2}\left(e_{11}+e_{22}-e_{12}+e_{21}\right), \\
a b\left(\frac{1-a^{4}}{2}\right) & =\frac{X}{2}\left(e_{11}-e_{22}-e_{12}-e_{21}\right), \\
a^{2} b\left(\frac{1-a^{4}}{2}\right) & =e_{21}+e_{12}, \\
a^{3} b\left(\frac{1-a^{4}}{2}\right) & =\frac{X}{2}\left(e_{11}-e_{22}+e_{12}+e_{21}\right) .
\end{aligned}
$$

Next we show how $\mathbf{Z G}\left(1-a^{4}\right)$ is embedded in $M_{2.2}(\mathbf{Q}[\sqrt{-2}])$. Any element in $\mathbf{Z} G\left(1-a^{4}\right)$ can be written in the form

$$
\left(2 \alpha_{0}+2 \alpha_{1} a+2 \alpha_{2} a^{2}+2 \alpha_{3} a^{3}+2 \delta_{0} b+2 \delta_{1} a b+2 \delta_{2} a^{2} b+2 \delta_{3} a^{3} b\right)\left(\frac{1-a^{4}}{2}\right),
$$

where all $\alpha_{i}, \delta_{i} \in \mathbf{Z}$. Such an element corresponds to the matrix

$$
\begin{aligned}
A= & 2\left(\alpha_{01}\left[\begin{array}{ll}
1 & 0 \\
0 & 1
\end{array}\right]+\alpha_{1} \frac{X}{2}\left[\begin{array}{rr}
1 & 1 \\
-1 & 1
\end{array}\right]+\alpha_{2}\left[\begin{array}{rr}
0 & -1 \\
1 & 0
\end{array}\right]+\alpha_{3} \frac{X}{2}\left[\begin{array}{rr}
1 & -1 \\
1 & 1
\end{array}\right]\right. \\
& \left.+\delta_{01}\left[\begin{array}{rr}
1 & 0 \\
1 & -1
\end{array}\right]+\delta_{1} \frac{X}{2}\left[\begin{array}{rr}
1 & -1 \\
-1 & -1
\end{array}\right]+\delta_{2}\left[\begin{array}{ll}
0 & 1 \\
1 & 0
\end{array}\right]+\delta_{3} \frac{X}{2}\left[\begin{array}{rr}
1 & 1 \\
1 & -1
\end{array}\right]\right) \\
= & \left.\begin{array}{cc}
2\left(\alpha_{0}+\delta_{0}\right) & 2\left(-\alpha_{2}+\delta_{2}\right) \\
+X\left(\alpha_{1}+\alpha_{3}+\delta_{1}+\delta_{3}\right) & +X\left(\alpha_{1}-\alpha_{3}-\delta_{1}+\delta_{3}\right) \\
2\left(\alpha_{2}+\delta_{2}\right) & 2\left(\alpha_{0}-\delta_{0}\right) \\
+X\left(-\alpha_{1}+\alpha_{3}-\delta_{1}+\delta_{3}\right) & +X\left(\alpha_{1}+\alpha_{3}-\delta_{1}-\delta_{3}\right)
\end{array}\right] .
\end{aligned}
$$

It is convenient to conjugate $A$ by the matrix $\left[\begin{array}{cc}1 & 1+X \\ 0 & 1\end{array}\right]$, obtaining

$$
\left[\begin{array}{lc}
2\left(\alpha_{0}-\alpha_{1}-\alpha_{2}+\alpha_{3}\right. & 4\left(-\alpha_{1}+\alpha_{3}+\delta_{0}-2 \delta_{1}+\delta_{2}\right) \\
\left.\quad+\delta_{0}-\delta_{1}-\delta_{2}+\delta_{3}\right) & +4 X\left(-\alpha_{2}+\delta_{0}-\delta_{2}+\delta_{3}\right) \\
+2 X\left(\alpha_{1}-\alpha_{2}+\delta_{1}-\delta_{2}\right) & \\
2\left(\alpha_{2}+\delta_{2}\right)+X\left(-\alpha_{1}+\alpha_{3}-\delta_{1}+\delta_{3}\right) & 2\left(\alpha_{0}+\alpha_{1}+\alpha_{2}-\alpha_{3}-\delta_{0}+\delta_{1}+\delta_{2}-\delta_{3}\right) \\
& +2 X\left(\alpha_{2}+\alpha_{3}-\delta_{1}+\delta_{2}\right)
\end{array}\right] .
$$


This is a matrix of the type

$$
\left[\begin{array}{cc}
2\left(m+m^{\prime} X\right) & 4\left(n+n^{\prime} X\right) \\
2 e+e^{\prime} X & 2\left(f+f^{\prime} X\right)
\end{array}\right]
$$

where $m, m^{\prime}, n, n^{\prime}, e, e^{\prime}, f, f^{\prime} \in \mathbf{Z}$.

Solving a system of 8 linear equations in 8 unknowns, we discover that a matrix of the above type comes from an element in $\mathbf{Z} G\left(1-\alpha^{4}\right)$ if and only if $m-f$ is divisible by 2 . We conclude that

$$
\begin{array}{r}
\mathbf{Z} G\left(1-a^{4}\right) \cong\left\{\left[\begin{array}{cc}
2\left(m+m^{\prime} \sqrt{-2}\right) & 4\left(n+n^{\prime} \sqrt{-2}\right) \\
2 e+e^{\prime} \sqrt{-2} & 2\left(f+f^{\prime} \sqrt{-2}\right)
\end{array}\right] \mid\right. \\
\left.m, m^{\prime}, n, n^{\prime}, e, e^{\prime}, f, f^{\prime} \in \mathbf{Z} \text {, and } 2 \mid(m-f)\right\} .
\end{array}
$$

Now, an element in $\mathbf{Z} G\left(1-a^{4}\right)$ is in $\Delta_{\mathbf{Z}}(G)\left(1-a^{4}\right)$ if and only if it can be written in the form

$$
\left(\alpha_{0}+\alpha_{1} a+\alpha_{2} a^{2}+\alpha_{3} a^{3}+\delta_{0} b+\delta_{1} a b+\delta_{2} a^{2} b+\delta_{3} a^{3} b\right)\left(1-a^{4}\right),
$$

where $\alpha_{0}+\alpha_{1}+\alpha_{2}+\alpha_{3}+\delta_{0}+\delta_{1}+\delta_{2}+\delta_{3}$ is divisible by 2 . By the previous work, it is easy to see that these elements correspond exactly to matrices of the form

$$
\left[\begin{array}{cc}
2\left(m+m^{\prime} \sqrt{-2}\right) & 4\left(n+n^{\prime} \sqrt{-2}\right) \\
2 e+e^{\prime} \sqrt{-2} & 2\left(f+f^{\prime} \sqrt{-2}\right)
\end{array}\right]
$$

where $2 \mid m$ and $2 \mid f$, i.e.

$$
\left(\Delta_{\mathbf{Z}}(G)\right)\left(1-a^{4}\right) \cong\left\{\left[\begin{array}{cc}
4 m+2 m^{\prime} \sqrt{-2} & 4 n+4 n^{\prime} \sqrt{-2} \\
2 e+e^{\prime} \sqrt{-2} & 4 f+2 f^{\prime} \sqrt{-2}
\end{array}\right] \mid m, m^{\prime}, n, n^{\prime}, e, e^{\prime}, f, f^{\prime} \in \mathbf{Z}\right\} .
$$

We are now ready to describe $\mathcal{U}(\mathbf{Z} G)$.

THEOREM 2. In $\mathcal{U}_{1}\left(\mathbf{Z} D_{16}^{-}\right), D_{16}^{-}$has a torsion-free normal complement which is the semi-direct product of the matrix group

$$
\begin{array}{r}
\left\{A=\left[\begin{array}{cr}
1+4 m+2 m^{\prime} \sqrt{-2} & 4 n+4 n^{\prime} \sqrt{-2} \\
2 e+e^{\prime} \sqrt{-2} & 1+4 f+2 f^{\prime} \sqrt{-2}
\end{array}\right] \mid\right. \\
\left.m, m^{\prime}, n, n^{\prime}, e, e^{\prime}, f, f^{\prime} \in \mathbf{Z}, \text { and } \operatorname{det}(a)=1\right\}
\end{array}
$$

by a free group of rank 3 .

Proof. We again set $G=D_{16}^{-}$. Let $V=\left\{u=1+\alpha\left(1-a^{2}\right) \mid u\right.$ a unit, $\left.\alpha \in \Delta_{\mathbf{Z}}(G)\right\}$. It is not hard to prove, and also well known [2], that $V$ is a torsion-free normal complement for $G$ in $U_{1}(\mathbf{Z} G)$. Under the natural epimorphism $f: \mathbf{Z} G \rightarrow \mathbf{Z} D_{\mathbf{8}}$ (mapping $a^{4}$ to 1 ), $V$ is mapped into the group $W$ mentioned in Theorem 1. Further $W$ is freely generated by the three bicyclic units $x_{1}=1+(1-b) a(1+b), x_{2}=1+(1-a b) a(1+a b)$ and $x_{3}=1+\left(1-a^{2} b\right) a\left(1+a^{2} b\right)$. 
Note that $x_{1}$ and $x_{3}$ are also bicyclic units when viewed as elements of $\mathbf{Z G}$. In $\mathbf{Z G}$,

and

$$
1+(1-b) a(1+b)=1+(a-b a)\left(1-a^{2}\right)
$$

$$
1+\left(1-a^{2} b\right) a\left(1+a^{2} b\right)=1+\left(a-b a^{7}\right)\left(1-a^{2}\right) .
$$

So both of these elements are in $V$.

Although $x_{2}$ is not the image of a bicyclic unit in $\mathbf{Z} G$, it still turns out that there is a unit

$$
u=1+\left(-1+a-a^{2}+a^{2} b\right)\left(1-a^{2}\right)+\left(2+a-a^{2}-a^{3}-b-a b+a^{2} b+a^{3} b-a^{4}\right)\left(1-a^{4}\right)
$$

in $V$ (with inverse

$$
\left.u^{-1}=1+\left(1-a+a^{2}-a^{2} b\right)\left(1-a^{2}\right)+\left(-a+a^{2}+a^{3}+b+a b-a^{2} b-a^{3} b-a^{4}\right)\left(1-a^{4}\right)\right)
$$

such that $f(u)=x_{2}$. We are able to see this because of the constructive isomorphism between $\left(\Delta_{\mathbf{Z}}(G)\right)\left(1-a^{4}\right)$ and a set of matrices.

Hence $f(V)=W$, and the freeness of $W$ tells us that $V$ is then the semi-direct product of $\{v \in V \mid f(v)=1\}$ by a free group of rank 3 .

Now $\{v \in V \mid f(v)=1\}$ is the set of units which can be written in the form $1+\alpha\left(1-a^{2}\right), \alpha \in \Delta_{\mathbf{Z}}(G)$, and also in the form $1+\beta\left(1-a^{4}\right), \beta \in \mathbf{Z G}$. This means that $\alpha-\beta\left(1+a^{2}\right)=\gamma\left(1+a^{2}+a^{4}+a^{6}\right)$ for some $\gamma \in \mathbf{Z G}$. Therefore the sum of the coefficients of $\beta$ is divisible by 2 . Since $\left(\beta+\delta\left(1+a^{4}\right)\right)\left(1-a^{4}\right)=\beta\left(1-a^{4}\right)$ for any $\delta \in \mathbf{Z} G$, we can assume that $\beta \in \Delta_{\mathbf{Z}}(G)$. Our previous remarks then yield that $\{v \in V \mid f(v)=1\}$ is faithfully represented by invertible matrices of the form

$$
\left[\begin{array}{cc}
1+4 m+2 m^{\prime} \sqrt{-2} & 4 n+4 n^{\prime} \sqrt{-2} \\
2 e+e^{\prime} \sqrt{-2} & 1+4 f+2 f^{\prime} \sqrt{-2}
\end{array}\right]
$$

where $m, m^{\prime}, n, n^{\prime}, e, e^{\prime}, f, f^{\prime} \in \mathbf{Z}$.

Since the only units in $\mathbf{Z}[\sqrt{-2}]$ are \pm 1 , the form of the given matrices forces the determinant to be 1 . We note, however, that any such matrix of determinant 1 has an inverse matrix of the same type, and so must represent a unit of the form $1+\beta\left(1-a^{4}\right)$ where $\beta \in \Delta_{\mathbf{Z}}(G)$.

The proof of the theorem is complete.

2. Description of $\mathcal{U}\left(\mathbf{Z} D_{16}\right)$. In this section we describe $\mathcal{U}(\mathbf{Z} G)$, where

$$
G=D_{16}=\left\langle a, b \mid a^{8}=b^{2}=1, b a=a^{7} b\right\rangle .
$$

The argument follows the same pattern as with $D_{16}^{-}$.

First we look again closely to the Wedderburn decomposition. Let $X=\left(a-a^{3}\right) \frac{1}{2}\left(1-a^{4}\right)$. Since $X$ is central in $Q G\left(\frac{1}{2}\left(1-a^{4}\right)\right)$, and $X^{2}=2\left(\frac{1}{2}\left(1-a^{4}\right)\right)$, $\mathbf{Q}\left[\left(a-a^{3}\right) \frac{1}{2}\left(1-a^{4}\right)\right]$ is a field isomorphic to $\mathbf{Q}[\sqrt{2}]$. We have $\mathbf{Q} G\left(\frac{1}{2}\left(1-a^{4}\right)\right) \cong$ $M_{2,2}(\mathbf{Q}[\sqrt{2}])$ and the following elements form an elementary matrix basis for $\mathbf{Q} G\left(\frac{1}{2}\left(1-a^{4}\right)\right)$ over $\mathbf{Q}\left[\left(a-a^{3}\right) \frac{1}{2}\left(1-a^{4}\right)\right]$ :

$$
\begin{array}{ll}
e_{11}=\left(\frac{1+b}{2}\right)\left(\frac{1-a^{4}}{2}\right), & e_{12}=-a^{2}\left(\frac{1-b}{2}\right)\left(\frac{1-a^{4}}{2}\right), \\
e_{21}=a^{2}\left(\frac{1+b}{2}\right)\left(\frac{1-a^{4}}{2}\right), & e_{22}=\left(\frac{1-b}{2}\right)\left(\frac{1-a^{4}}{2}\right) .
\end{array}
$$


In $\mathbf{Q} G\left(\frac{1}{2}\left(1-a^{4}\right)\right)$, we have

$$
\begin{aligned}
a\left(\frac{1-a^{4}}{2}\right) & =\frac{X}{2}\left(e_{11}+e_{22}-e_{12}+e_{21}\right), \\
b\left(\frac{1-a^{4}}{2}\right) & =e_{11}-e_{22}, \\
a^{2}\left(\frac{1-a^{4}}{2}\right) & =-e_{12}+e_{21}, \\
a^{3}\left(\frac{1-a^{4}}{2}\right) & =\frac{X}{2}\left(-e_{11}-e_{22}-e_{12}+e_{21}\right), \\
a b\left(\frac{1-a^{4}}{2}\right) & =\frac{X}{2}\left(e_{11}-e_{22}+e_{12}+e_{21}\right), \\
a^{2} b\left(\frac{1-a^{4}}{2}\right) & =e_{12}+e_{21}, \\
a^{3} b\left(\frac{1-a^{4}}{2}\right) & =\frac{X}{2}\left(-e_{11}+e_{22}+e_{12}+e_{21}\right) .
\end{aligned}
$$

Next we show how $\mathbf{Z} G\left(1-a^{4}\right)$ is embedded in $M_{2.2}(\mathbf{Q}[\sqrt{2}])$. An element in $\mathbf{Z} G\left(1-a^{4}\right)$ can be written in the form

$$
\left(2 \alpha_{0}+2 \alpha_{1} a+2 \alpha_{2} a^{2}+2 \alpha_{3} a^{3}+2 \delta_{0} b+2 \delta_{1} a b+2 \delta_{2} a^{2} b+2 \delta_{3} a^{3} b\right)\left(\frac{1-a^{4}}{2}\right),
$$

where all $\alpha_{i}, \delta_{i} \in \mathbf{Z}$. Such an element corresponds to the matrix

$$
\begin{aligned}
A= & 2\left(\alpha_{0}\left[\begin{array}{ll}
1 & 0 \\
0 & 1
\end{array}\right]+\alpha_{1} \frac{X}{2}\left[\begin{array}{rr}
1 & -1 \\
1 & 1
\end{array}\right]+\alpha_{2}\left[\begin{array}{rr}
0 & -1 \\
1 & 0
\end{array}\right]+\alpha_{3} \frac{X}{2}\left[\begin{array}{rr}
-1 & -1 \\
1 & -1
\end{array}\right]\right. \\
& \left.+\delta_{0}\left[\begin{array}{rr}
1 & 0 \\
0 & -1
\end{array}\right]+\delta_{1} \frac{X}{2}\left[\begin{array}{rr}
1 & 1 \\
1 & -1
\end{array}\right]+\delta_{2}\left[\begin{array}{ll}
0 & 1 \\
1 & 0
\end{array}\right]+\delta_{3} \frac{X}{2}\left[\begin{array}{rr}
-1 & 1 \\
1 & 1
\end{array}\right]\right) .
\end{aligned}
$$

Conjugating $A$ by $\left[\begin{array}{cc}1 & 1+X \\ 0 & 1\end{array}\right]$, we obtain

$$
\left[\begin{array}{cc}
2\left(\alpha_{0}-\alpha_{1}-\alpha_{2}-\alpha_{3}+\delta_{0}-\delta_{1}\right. & 4\left(-\alpha_{1}-2 \alpha_{2}-\alpha_{3}+\delta_{0}-\delta_{2}-2 \delta_{3}\right) \\
\left.-\delta_{2}-\delta_{3}\right)+2 X\left(-\alpha_{2}-\alpha_{3}-\delta_{2}-\delta_{3}\right) & +4 X\left(-\alpha_{1}-\alpha_{2}-\alpha_{3}+\delta_{0}-\delta_{2}-\delta_{3}\right) \\
2\left(\alpha_{2}+\delta_{2}\right) & 2\left(\alpha_{0}+\alpha_{1}+\alpha_{2}+\alpha_{3}-\delta_{0}+\delta_{1}\right. \\
+X\left(\alpha_{1}+\alpha_{3}+\delta_{1}+\delta_{3}\right) & \left.+\delta_{2}+\delta_{3}\right)+2 X\left(\alpha_{1}+\alpha_{2}+\delta_{2}+\delta_{3}\right)
\end{array}\right]
$$

This is a matrix of the type

$$
\left[\begin{array}{cc}
2\left(m+m^{\prime} X\right) & 4\left(n+n^{\prime} X\right) \\
2 e+e^{\prime} X & 2\left(f+f^{\prime} X\right)
\end{array}\right]
$$

where $m, m^{\prime}, n, n^{\prime}, e, e^{\prime}, f, f^{\prime} \in \mathbf{Z}$. 
Solving a system of 8 linear equations in 8 unknowns, we find that a matrix of the above type comes from an element of $\mathbf{Z} G\left(1-a^{4}\right)$ if and only if $m-f$ is divisible by 2 . We conclude that

$$
\begin{array}{r}
\mathbf{Z} G\left(1-a^{4}\right) \cong\left\{\left[\begin{array}{cr}
2\left(m+m^{\prime} \sqrt{2}\right) & 4\left(n+n^{\prime} \sqrt{2}\right) \\
2 e+e^{\prime} \sqrt{2} & 2\left(f+f^{\prime} \sqrt{2}\right)
\end{array}\right] \mid\right. \\
\left.m, m^{\prime}, n, n^{\prime}, e, e^{\prime}, f, f^{\prime} \in \mathbf{Z}, \text { and } 2 \mid(m-f)\right\} .
\end{array}
$$

Exactly as with $D_{16}^{-}$, we then deduce that

$$
\left(\Delta_{\mathbf{Z}}(G)\right)\left(1-a^{4}\right) \cong\left\{\left[\begin{array}{cc}
4 m+2 m^{\prime} \sqrt{2} & 4 n+4 n^{\prime} \sqrt{2} \\
2 e+e^{\prime} \sqrt{2} & 4 f+2 f^{\prime} \sqrt{2}
\end{array}\right] \mid m, m^{\prime}, n, n^{\prime}, e, e^{\prime}, f, f^{\prime} \in \mathbf{Z}\right\} .
$$

Let $V$ be the set of units in $\mathbf{Z} G$ of the form $1+\alpha\left(1-a^{2}\right), \alpha \in \Delta_{\mathbf{Z}}(G)$. Again, $V$ is a torsion-free normal complement for $G$ in $\mathcal{U}_{1}(\mathbf{Z} G)$, and if $f: \mathbf{Z} G \rightarrow \mathbf{Z} D_{\mathrm{8}}$ is the natural epimorphism, $f(V) \subseteq W$, where $W$ is the group determined in Theorem 1 . But all of the bicyclic units in $\mathbf{Z} D_{8}$ are images of bicyclics in $\mathbf{Z G}$, and these bicyclics are, in fact, in $V$. Hence $f(V)=W$ follows from Theorem 1 , and the freeness of $W$ tells us that $V$ is the semi-direct product of $\{v \in V \mid f(v)=1\}$ by a free group of rank 3 .

As before, we see that $\{v \in V \mid f(v)=1\}$ is precisely the set of all units which can be written in the form $1+\beta\left(1-a^{4}\right), \beta \in \Delta_{\mathbf{Z}}(G)$. Our previous remarks tell us that this group is faithfully represented in invertible matrices of the form

$$
\left[\begin{array}{cc}
1+4 m+2 m^{\prime} \sqrt{2} & 4 n+4 n^{\prime} \sqrt{2} \\
2 e+e^{\prime} \sqrt{2} & 1+4 f+2 f^{\prime} \sqrt{2}
\end{array}\right]
$$

where $m, m^{\prime}, n, n^{\prime}, e, e^{\prime}, f, f^{\prime} \in \mathbf{Z}$.

Recall now that the units of $\mathbf{Z}[\sqrt{2}]$ are precisely those elements $s+t \sqrt{2}$, with $s, t \in \mathbf{Z}$, for which $s^{2}-2 t^{2}= \pm 1$. A matrix of the type we are considering has determinant

$$
\begin{aligned}
(1+ & \left.4 m+2 m^{\prime} \sqrt{2}\right)\left(1+4 f+2 f^{\prime} \sqrt{2}\right)-\left(4 n+4 n^{\prime} \sqrt{2}\right)\left(2 e+e^{\prime} \sqrt{2}\right) \\
= & 1+4 m+4 f+16 m f+8 m^{\prime} f^{\prime}-8 n e-8 n^{\prime} e^{\prime} \\
& +\left(2 f^{\prime}+8 m f^{\prime}+2 m^{\prime}+8 m^{\prime} f-4 n e^{\prime}-4 n^{\prime} e\right) \sqrt{2} .
\end{aligned}
$$

For such an element to be a unit $s+t \sqrt{2}$, we note that $s^{2}-2 t^{2}=1$ is the only possibility. We also note that $s \equiv 1(\bmod 4)$.

Recall further that the units of $\mathbf{Z}[\sqrt{2}]$ consist precisely of the elements $\pm \lambda^{p}, p \in \mathbf{Z}$, where $\lambda=1+\sqrt{2}$ (cf. [7]). Such a unit satisfies $s^{2}-2 t^{2}=1$ and $s=1(\bmod 4)$ if and only if it is in the cyclic subgroup generated by $-\lambda^{2}=-3-2 \sqrt{2}$ (or equivalently, its inverse $-3+2 \sqrt{2})$.

Finally, note that if we take a matrix $B$ of the form

$$
\left[\begin{array}{cc}
1+4 m+2 m^{\prime} \sqrt{2} & 4 n+4 n^{\prime} \sqrt{2} \\
2 e+e^{\prime} \sqrt{2} & 1+4 f+2 f^{\prime} \sqrt{2}
\end{array}\right]
$$

then $(-3-2 \sqrt{2}) B$ and $(-3+2 \sqrt{2}) B$ are of the same form. We conclude that the inverse of any such matrix is of the same form, and hence all of these matrices represent units of the type $1+\beta\left(1-a^{4}\right)$, where $\beta \in \Delta_{\mathbf{z}}(G)$. 
We have proved the following theorem.

THEOREM 3. In $U_{1}\left(\mathbf{Z} D_{16}\right), D_{16}$ has a torsion-free normal complement which is the semi-direct product of the matrix group

$$
\left\{A=\left[\begin{array}{cc}
1+4 m+2 m^{\prime} \sqrt{2} & 4 n+4 n^{\prime} \sqrt{2} \\
2 e+e^{\prime} \sqrt{2} & 1+4 f+2 f^{\prime} \sqrt{2}
\end{array}\right] \mid\right.
$$

$$
\left.m, m^{\prime}, n, n^{\prime}, e, e^{\prime}, f, f^{\prime} \in \mathbf{Z}, \text { and } \operatorname{det}(A) \in\langle-3-2 \sqrt{2}\rangle\right\}
$$

by a free group of rank 3.

3. Description of $U\left(\mathbf{Z} Q_{16}\right)$. Now we describe $U(\mathbf{Z} G)$, where

$$
G=Q_{16}=\left\langle a, b \mid a^{8}=1, a^{4}=b^{2}, b a=a^{7} b\right\rangle .
$$

As before it is easy to see that $Z\left(\mathbf{Q} G\left(\frac{1}{2}\left(1-a^{4}\right)\right)\right)=\mathbf{Q}[X]$ is a field isomorphic to $\mathbf{Q}[\sqrt{2}]$, where $X=\left(a-a^{3}\right) \frac{1}{2}\left(1-a^{4}\right)$ is such that $X^{2}=2\left(\frac{1}{2}\left(1-a^{4}\right)\right)$. Further if we let $1=\frac{1}{2}\left(1-a^{4}\right)$, $\mathbf{i}=a^{2} \frac{1}{2}\left(1-a^{4}\right), \quad \mathbf{j}=b \frac{1}{2}\left(1-a^{4}\right), \quad \mathbf{k}=a^{2} b \frac{1}{2}\left(1-a^{4}\right), \quad$ then one easily verifies that $\mathbf{Q} G\left(\frac{1}{2}\left(1-a^{4}\right)\right.$ ) is a quaternion algebra over $\mathbf{Q}[X]$ (with basis $\mathbf{1}, \mathbf{i}, \mathbf{j}, \mathbf{k}$ ). In $\mathbf{Q} G\left(\frac{1}{2}\left(1-a^{4}\right)\right)$ we have:

$$
\begin{aligned}
a\left(\frac{1-a^{4}}{2}\right) & =\frac{1}{2}(X \mathbf{1}+X \mathbf{i}), \\
a^{2}\left(\frac{1-a^{4}}{2}\right) & =\mathbf{i}, \\
a^{3}\left(\frac{1-a^{4}}{2}\right) & =\frac{1}{2}(-X \mathbf{1}+X \mathbf{i}), \\
b\left(\frac{1-a^{4}}{2}\right) & =\mathbf{j}, \\
a b\left(\frac{1-a^{4}}{2}\right) & =\frac{1}{2}(X \mathbf{j}+X \mathbf{k}), \\
a^{2} b\left(\frac{1-a^{4}}{2}\right) & =\mathbf{k}, \\
a^{3} b\left(\frac{1-a^{4}}{2}\right) & =\frac{1}{2}(-X \mathbf{j}+X \mathbf{k}) .
\end{aligned}
$$

Next we look closely to the embedding of $\mathbf{Z} G\left(1-a^{4}\right)$ in $\mathbf{Q} G\left(\frac{1}{2}\left(1-a^{4}\right)\right) \cong \mathbf{H}(\mathbf{Q}[\sqrt{2}])$. An element in $\mathbf{Z G}\left(1-a^{4}\right)$ can be written in the form

$$
\begin{aligned}
2\left(\alpha_{0}\right. & \left.+\alpha_{1} a+\alpha_{2} a^{2}+\alpha_{3} a^{3}+\delta_{0} b+\delta_{1} a b+\delta_{2} a^{2} b+\delta_{3} a^{3} b\right)\left(\frac{1-a^{4}}{2}\right) \\
= & 2 \alpha_{0} \mathbf{1}+\alpha_{1}(X \mathbf{1}+X \mathbf{i})+2 \alpha_{2} \mathbf{i}+\alpha_{3}(-X \mathbf{1}+X \mathbf{i}) \\
& +2 \delta_{0} \mathbf{j}+\delta_{1}(X \mathbf{j}+X \mathbf{k})+2 \delta_{2} \mathbf{k}+\delta_{3}(-X \mathbf{j}+X \mathbf{k}) \\
= & \left(2 \alpha_{0}+\left(\alpha_{1}-\alpha_{3}\right) X\right) \mathbf{1}+\left(2 \alpha_{2}+\left(\alpha_{1}+\alpha_{3}\right) X\right) \mathbf{i} \\
& +\left(2 \delta_{0}+\left(\delta_{1}-\delta_{3}\right) X\right) \mathbf{j}+\left(2 \delta_{2}+\left(\delta_{1}+\delta_{3}\right) X\right) \mathbf{k}
\end{aligned}
$$


for some $\alpha_{0}, \alpha_{1}, \alpha_{2}, \alpha_{3}, \delta_{0}, \delta_{1}, \delta_{2}, \delta_{3} \in \mathbf{Z}$. Such an element corresponds to a quaternion element of the form

$$
(2 s+t \sqrt{2}) \mathbf{1}+(2 c+d \sqrt{2}) \mathbf{i}+(2 e+f \sqrt{2}) \mathbf{j}+(2 m+n \sqrt{2}) \mathbf{k}
$$

Solving a system of 8 linear equations in 8 unknowns it follows that a quaternion element of the above type comes from an element of $\mathbf{Z G}\left(1-a^{4}\right)$ if and only if 2 divides $t+d$ and 2 divides $f+n$. It follows that $\Delta_{\mathbf{Z}}(G)\left(1-a^{4}\right)$ is isomorphic with

$$
\begin{array}{r}
\{(2 s+t \sqrt{2}) \mathbf{1}+(2 c+d \sqrt{2}) \mathbf{i}+(2 e+f \sqrt{2}) \mathbf{j}+(2 m+n \sqrt{2}) \mathbf{k}|2|(t+d), \\
2 \mid(f+n) \text { and } 2 \mid(s+c+d+e+m+n)\} .
\end{array}
$$

Let $V$ be the set of all units of $\mathbf{Z} G$ of the form $1+\alpha\left(1-a^{2}\right), \alpha \in \Delta_{\mathbf{Z}}(G)$. Again, $V$ is a torsion-free normal complement for $G$ in $U_{1}(\mathbf{Z G})$. Under the natural epimorphism $f: \mathbf{Z} G \rightarrow \mathbf{Z} D_{\mathbf{8}}$ (mapping $a^{4}$ to 1 ), $V$ is mapped into the group $W$ mentioned in Theorem 1 . Recall that $W$ is generated by

$$
\begin{aligned}
& x_{1}=1+(1-b) a(1+b)=1+\left(a+a b-a^{3} b-a^{3}\right), \\
& x_{2}=1+(1-a b) a(1+a b)=1+\left(a+a^{2} b-b-a^{3}\right), \\
& x_{3}=1+\left(1-a^{2} b\right) a\left(1+a^{2} b\right)=1+\left(a+a^{3} b-a b-a^{3}\right) .
\end{aligned}
$$

Note that any unit $u=1+2 \alpha\left(1-a^{2}\right) \in W$ is an image of a unit in $V$, namely of $1+\alpha\left(1-a^{2}\right)\left(1+a^{4}\right)$. Also $W_{2}=\left\{u=1+2 \alpha\left(1-a^{2}\right) \mid u \in \mathcal{U}\left(\mathbf{Z} D_{8}\right)\right\}$ is a normal subgroup of $W$ and $W / W_{2}$ is an abelian group of order 4 (note that $x_{1} x_{3}^{-1} \in W_{2}$ ). Hence $f(V)$ is of finite index 1,2 or 4 in $W$. We claim it is of index 4 and thus $f(V)=W_{2}$. For this it is sufficient to show that $x_{1} \notin f(V), x_{2} \notin f(V)$ and $x_{1} x_{2} \notin f(V)$.

We will constantly use the fact that the only units in the quaternion algebra with coefficients in $\mathbf{Z}[\sqrt{2}]$ are of the form $\alpha \mathbf{1}, \alpha \mathbf{i}, \alpha \mathbf{j}$ and $\alpha \mathbf{k}$, where $\alpha$ is a unit in $\mathbf{Z}[\sqrt{2}]$. Assume first that $x_{1} \in f(V)$. In other words, there exists a unit $u$ in $\mathbf{Z} G$ such that $u=1+\left(a+a b-a^{3} b-a^{3}\right)+\alpha\left(1-a^{4}\right)$ for some $\alpha \in \mathbf{Z} G$. Note that

$$
u^{-1}=1-\left(a+a b-a^{3} b-a^{3}\right)+\beta\left(1-a^{4}\right)
$$

for some $\beta \in \mathbf{Z G}$. Now $u\left(\frac{1}{2}\left(1-a^{4}\right)\right)$ is a unit in $\mathbf{Z} G\left(\frac{1}{2}\left(1-a^{4}\right)\right)$, and the element of the quaternion algebra corresponding to it is of the form

$$
\begin{aligned}
& 1+\frac{1}{2}(\sqrt{2} \mathbf{1}+\sqrt{2} \mathbf{i})+\frac{1}{2}(\sqrt{2} \mathbf{j}+\sqrt{2} \mathbf{k})-\frac{1}{2}(-\sqrt{2} \mathbf{j}+\sqrt{2} \mathbf{k})-\frac{1}{2}(-\sqrt{2} \mathbf{1}+\sqrt{2} \mathbf{i}) \\
& +(2 s+t \sqrt{2}) \mathbf{1}+(2 c+d \sqrt{2}) \mathbf{i}+(2 e+f \sqrt{2}) \mathbf{j}+(2 m+n \sqrt{2}) \mathbf{k},
\end{aligned}
$$

where $s, t, c, d, e, f, m, n \in \mathbf{Z}, 2 \mid(t+d)$ and $2 \mid(f+n)$. This element can be written as

$$
(1+2 s+(t+1) \sqrt{2}) \mathbf{1}+(2 c+d \sqrt{2}) \mathbf{i}+(2 e+(f+1) \sqrt{2}) \mathbf{j}+(2 m+n \sqrt{2}) \mathbf{k} .
$$

Since the inverse of this element corresponds to $u^{-1}\left(\frac{1}{2}\left(1-a^{4}\right)\right)$ and also has all coefficients in $\mathbf{Z}[\sqrt{2}]$, we conclude that $1+2 s+(t+1) \sqrt{2}$ must be a unit in $\mathbf{Z}[\sqrt{2}]$ and $2 c=d=2 e=$ $f+1=2 m=n=0$. This contradicts $2 \mid(f+n)$, so we conclude that $x_{1} \notin f(V)$.

Next assume $x_{2} \in f(V)$, so there exists a unit $u$ in $\mathbf{Z} G$ such that

$$
u=1+\left(a+a^{2} b-b-a^{3}\right)+\alpha\left(1-a^{4}\right)
$$

for some $\alpha \in \mathbf{Z} G$. Note that $u^{-1}=1-\left(a+a^{2} b-b-a^{3}\right)+\beta\left(1-a^{4}\right)$. In this case, $u\left(\frac{1}{2}\left(1-a^{4}\right)\right)$ corresponds to the quaternion algebra element

$$
(1+2 s+(t+1) \sqrt{2}) \mathbf{1}+(2 c+d \sqrt{2}) \mathbf{i}+(2 e-1+f \sqrt{2}) \mathbf{j}+(2 m+1+n \sqrt{2}) \mathbf{k}
$$


Since the coefficients of $u^{-1}\left(\frac{1}{2}\left(1-a^{4}\right)\right)$ are also in $\mathbf{Z}[\sqrt{2}]$, we conclude that either $1+2 s=0$ or $2 e-1=0$, a contradiction. Hence $x_{2} \notin f(V)$.

Finally, assume that $x_{1} x_{2} \in f(V)$. Now

$$
\begin{aligned}
x_{1} x_{2} & =\left(1+\left(a+a b-a^{3} b-a^{3}\right)\right)\left(1+\left(a+a^{2} b-b-a^{3}\right)\right) \\
& =1+(-2+b-a b)\left(1-a^{2}\right) .
\end{aligned}
$$

So we conclude that there is a unit $u$ in $\mathbf{Z} G$ of the form $u=1+(-2+b-a b)\left(1-a^{2}\right)+$ $\alpha\left(1-a^{4}\right)$ for some $\alpha \in \mathbf{Z} G$. Also $\left(x_{1} x_{2}\right)^{-1}=1+(-2-b+a b)\left(1-a^{2}\right)$, so $u^{-1}$ is of the form $u^{-1}=1+(-2-b+a b)\left(1-a^{2}\right)+\beta\left(1-a^{4}\right)$. In this case, $u\left(\frac{1}{2}\left(1-a^{4}\right)\right)$ corresponds to the element

$$
(-1+2 s+t \sqrt{2}) \mathbf{1}+(2(c+1)+d \sqrt{2}) \mathbf{i}+(2 e+1+f \sqrt{2}) \mathbf{j}+(2 m-1+(n-1) \sqrt{2}) \mathbf{k} .
$$

Since the coefficients of $u^{-1}\left(\frac{1}{2}\left(1-a^{4}\right)\right)$ are also in $\mathbf{Z}[\sqrt{2}]$, we conclude that either $-1+2 s=0$ or $2 e+1=0$, a contradiction. Hence $x_{1} x_{2} \notin f(V)$.

We have now shown that $f(V)$ is a subgroup of index 4 in a free group of rank 3 . Hence $f(V)$ is a free group of rank 9 .

THEOREM 4. In $U_{1}\left(\mathbf{Z} Q_{16}\right), Q_{16}$ has a torsion-free normal complement which is the direct product of an infinite cyclic group and a free group of rank 9.

Proof. By the previous work, it is clear that $f(V)$ is a free group of rank 9 and that $V$ is a semi-direct product of

$$
\{v \in V \mid f(v)=1\}=\left\{v=1+\alpha\left(1-a^{4}\right) \mid \alpha \in \Delta_{\mathbf{Z}}(G), v \text { a unit }\right\}
$$

by a free group of rank 9 . We also know that an element $1+\alpha\left(1-a^{4}\right), \alpha \in \Delta_{\mathbf{Z}}(G)$, is faithfully represented by a quaternion element of the form

$$
(1+2 s+t \sqrt{2}) \mathbf{1}+(2 c+d \sqrt{2}) \mathbf{i}+(2 e+f \sqrt{2}) \mathbf{j}+(2 m+n \sqrt{2}) \mathbf{k}
$$

where $2|(t+d), 2|(f+n)$ and $2 \mid(s+c+d+e+m+n)$. But as before, if such an element is a unit, we must have $1+2 s+t+\sqrt{2}$ a unit in $Z[\sqrt{2}]$ and $c=d=e=f=m=$ $n=0$. The conditions $2|s, 2| t$ say precisely that $1+2 s+t \sqrt{2}$ is in the cyclic subgroup generated by $-3+2 \sqrt{2}$. Since central elements of $\Delta_{\mathbf{Z}}(G)\left(1-a^{4}\right)$ are central in $\mathbf{Z} G$, the result follows.

4. Description of $\mathcal{U}\left(\mathbf{Z}\left(C_{2} \times D_{8}\right)\right)$. We consider $\mathcal{U}(\mathbf{Z} G)$, where $G=C_{2} \times D_{8}, C_{2}=$ $\{1, c\}$.

Let $W=\left\{u=1+\left(1-a^{2}\right) \alpha \mid \alpha \in \Delta_{\mathbf{z}}(G), u\right.$ a unit $\}$. Again by the result of Cliff, Sehgal and Weiss [2] (or directly), it follows that $W$ is a torsion-free normal complement for $G$ in $U_{1}(\mathbf{Z} G)$.

Let $f: \mathbf{Z} G \rightarrow \mathbf{Z} D_{8}$ be the natural epimorphism (mapping $c$ to 1 ). Because of Theorem $1, f(W)$ is a free group of rank 3 . It follows that $W$ is a semi-direct product of $K=\left\{u=1+\left(1-a^{2}\right)(1-c) \alpha \mid \alpha \in \mathbf{Z} G, u\right.$ a unit $\}$ by a free group of rank 3 . Hence we will study the structure of $K$.

The following form an elementary matrix basis for $\mathbf{Q} G\left(\frac{1}{2}\left(1-a^{2}\right)\right)\left(\frac{1}{2}(1-c)\right)$ over $\mathbf{Q}$ :

$$
\begin{array}{ll}
e_{11}=\left(\frac{1+b}{2}\right)\left(\frac{1-a^{2}}{2}\right)\left(\frac{1-c}{2}\right), & e_{12}=\left(\frac{a b-a}{2}\right)\left(\frac{1-a^{2}}{2}\right)\left(\frac{1-c}{2}\right), \\
e_{21}=\left(\frac{a b+a}{2}\right)\left(\frac{1-a^{2}}{2}\right)\left(\frac{1-c}{2}\right), & e_{22}=\left(\frac{1-b}{2}\right)\left(\frac{1-a^{2}}{2}\right)\left(\frac{1-c}{2}\right) .
\end{array}
$$


Note that

$$
\begin{aligned}
a\left(\frac{1-a^{2}}{2}\right)\left(\frac{1-c}{2}\right) & =e_{21}-e_{12} \\
b\left(\frac{1-a^{2}}{2}\right)\left(\frac{1-c}{2}\right) & =e_{11}-e_{22} \\
a b\left(\frac{1-a^{2}}{2}\right)\left(\frac{1-c}{2}\right) & =e_{12}+e_{21} .
\end{aligned}
$$

Next we look again more closely at the embedding of $\mathbf{Z} G\left(1-a^{2}\right)(1-c)$ in $\mathbf{Q} G\left(\frac{1}{2}\left(1-a^{2}\right)\right)\left(\frac{1}{2}(1-c)\right) \cong M_{2.2}(\mathbf{Q})$. A typical element of $\mathbf{Z} G\left(1-a^{2}\right)(1-c)$ is

$$
\left(\alpha_{0}+\alpha_{1} a+\alpha_{2} b+\alpha_{3} a b\right)\left(1-a^{2}\right)(1-c)=\left(4 \alpha_{0}+4 \alpha_{1} a+4 \alpha_{2} b+4 \alpha_{3} a b\right)\left(\frac{1-a^{2}}{2}\right)\left(\frac{1-c}{2}\right) \text {. }
$$

As a matrix, this is

$$
\left[\begin{array}{cc}
4\left(\alpha_{0}+\alpha_{2}\right) & 4\left(-\alpha_{1}+\alpha_{3}\right) \\
4\left(\alpha_{1}+\alpha_{3}\right) & 4\left(\alpha_{0}-\alpha_{2}\right)
\end{array}\right]
$$

Conjugating by $\left[\begin{array}{ll}1 & 1 \\ 0 & 1\end{array}\right]$, we obtain

$$
\left[\begin{array}{cc}
4\left(\alpha_{0}-\alpha_{1}+\alpha_{2}-\alpha_{3}\right) & 8\left(-\alpha_{1}+\alpha_{2}\right) \\
4\left(\alpha_{1}+\alpha_{3}\right) & 4\left(\alpha_{0}+\alpha_{1}-\alpha_{2}+\alpha_{3}\right)
\end{array}\right]
$$

These are all matrices of the form

$$
\left[\begin{array}{cc}
4 e & 8 f \\
4 c & 4 d
\end{array}\right]
$$

with $e, f, c, d \in \mathbf{Z}$.

By solving equations, we see that such a matrix is obtained from an element in $\mathbf{Z} G\left(1-a^{2}\right)(1-c)$ if and only if $2 \mid(e+d)$. So units in $K$ are faithfully represented by invertible matrices of the form

$$
\left[\begin{array}{cc}
1+4 e & 8 f \\
4 c & 1+4 d
\end{array}\right]
$$

with $2 \mid(e+d), e, f, \varepsilon, d \in \mathbf{Z}$. But such matrices must have determinant 1 , and this forces $2 \mid(e+d)$. Also the inverse of any such matrix is of the same type. Hence

$$
K \cong\left\{A=\left[\begin{array}{cc}
1+4 e & 8 f \\
4 c & 1+4 d
\end{array}\right] \mid e, f, c, d \in \mathbf{Z}, \operatorname{det}(A)=1\right\}
$$

This group is of index 2 in $\Gamma(4)$, the principal congruence subgroup of level 4. As $\Gamma(4)$ is a free group of rank 5 [5], $K$ is a free group of rank 9 . So we have proved the following theorem.

TheOREM 5. In $U_{1}\left(\mathbf{Z}\left(C_{2} \times D_{8}\right)\right), C_{2} \times D_{8}$ has a torsion-free normal complement which is a semi-direct product of a free group of rank 9 by a free group of rank 3 . 
5. Bicyclic and Bass cyclic units. We now turn our attention to the subgroup of $\mathcal{U}(\mathbf{Z G})$ generated by the Bass cyclic and bicyclic units. The following is a slight extension of Proposition 6.3 in [9].

THEOREM 6. Let $G$ be a finite 2-group and let $f: G \rightarrow D_{8}$ be an epimorphism. If at least 2 of $b, a b, a^{2} b, a^{3} b$ do not have preimages in $G$ of order 2 , then the Bass cyclic and bicyclic units in $\mathbf{Z G}$ do not generate a subgroup of finite index in $u(\mathbf{Z G})$.

Once this has been proved, we have settled the problem for all indecomposable groups of order 16 .

COROLlary 7. The Bass cyclic and bicyclic units do not generate a subgroup of finite index in $U(\mathrm{Z} G)$, where $G=P, Q_{16}, D_{16}^{-}$or $H$.

Proof of the theorem. We will abuse notation and also use $f$ to denote the ring epimorphism $\mathbf{Z} G \rightarrow \mathbf{Z} D_{\mathrm{8}}$ and the group homomorphism $\mathscr{U}(\mathbf{Z} G) \rightarrow \mathscr{U}\left(\mathbf{Z} D_{\mathrm{k}}\right)$.

Since $\mathbf{Z} D_{8}$ has no non-trivial Bass cyclic units, any Bass cyclic in $\mathbf{Z} G$ must map to a trivial unit in $\mathbf{Z} D_{8}$. In fact, it is not hard to see that all Bass cyclic units map to 1 in $\mathbf{Z} D_{8}$.

Next consider a bicyclic unit $\gamma=1+(1-g) h \hat{g}$ in $\mathbf{Z} G$, where $\hat{g}=1+g+\ldots+$ $g^{n-1}, n=o(g)$. Then either $f(\gamma)=1$ or

$$
f(\gamma)=1+c(1-f(g)) f(h) \widehat{f(g)}=(1+(1-f(g)) f(h) \widehat{f(g)})^{c},
$$

where $c=o(g) / o(f(g))$. The bicyclic units of $\mathbf{Z} D_{8}$ are $x_{1}=1+(1-b) a(1+b), x_{2}=$ $1+(1-a b) a(1+a b), x_{3}=1+\left(1-a^{2} b\right) a\left(1+a^{2} b\right)$ and $x_{4}=1+\left(1-a^{3} b\right) a\left(1+a^{3} b\right)$. Further $x_{4}=x_{3}^{-1} x_{2}^{-1} x_{1}^{-1}$. The given condition on $G$ yields that at least two of these bicyclic units are not images of bicyclic units in $\mathbf{Z G}$.

Let $B$ be the subgroup of $U(\mathbf{Z G})$ generated by the Bass cyclic and the bicyclic units. From the remarks above, and the fact that $G$ is a 2-group, it follows that $f(B)$ is a proper subgroup of $W$ (as described in Theorem 1) requiring at most 4 generators. Since $W$ is a free group of rank 3 , we conclude that $f(B)$ must be of infinite index in $W$.

Next recall (from Theorem 1) that $W$ is also the set of units in $Z D_{8}$ of the form $1+\alpha\left(1-a^{2}\right)$, where $\alpha \in \Delta_{\mathbf{z}}\left(D_{8}\right)$. Let $W_{i}$ be the subgroup of $W$ consisting of those units which can be written in the form $1+2^{i} \beta\left(1-a^{2}\right)$ for some $\beta \in \mathbf{Z} D_{8}$. Note that $W_{i} \subseteq W$ because $\left(2-\left(1-a^{2}\right)\right)\left(1+a^{2}\right)=2\left(1+a^{2}\right)$. Also note that for all $i, W_{i}$ is a normal subgroup of $W$ and that $W / W_{1}$ and $W_{i} / W_{i+1}$ are of exponent 2 and hence are abelian. Since $W$ is finitely generated, it follows that $W / W_{1}$ and all $W_{i} / W_{i+1}$ are finite, so $W / W_{i}$ is finite.

Let $K$ be the kernel of $f: G \rightarrow D_{8}$. We know that $|K|=2^{l}$ for some $l \geq 1$, and let $1+2^{\prime} \beta\left(1-a^{2}\right)$ be any element of $W_{l}$. Choose $a_{1}, \beta_{1}$ to be any preimages of $a, \beta$ in $\mathbf{Z G ,}$ and define $\hat{K}=\sum_{g \in K} g$. It is easy to see that $u=1+\hat{K} \beta_{1}\left(1-a_{1}^{2}\right)$ is a unit in $\mathbf{Z} G$ and that $f(u)=1+2^{l} \beta\left(1-a^{2}\right)$. Hence $W_{l} \subseteq f(\mathcal{U}(\mathbf{Z} G))$.

Now assume the contrary of the result we wish to prove, namely $B$ is of finite index in $\mathcal{U}(\mathbf{Z} G)$. It follows that $f(B)$ is of finite index in $f(\mathcal{U}(\mathbf{Z} G))$. Since $f(B) \subseteq W$, this yields $f(B)$ is of finite index in $f(\mathcal{U}(\mathbf{Z} G)) \cap W$. However it is shown above that the latter subgroup is of finite index in $W$, contradicting our earlier result that $f(B)$ is of infinite index in $W$. 


\section{REFERENCES}

1. H. Bass, The Dirichlet unit theorem, induced characters and Whitehead groups of finite groups, Topology 4 (1966), 391-410.

2. G. H. Cliff, S.K. Sehgal and A. R. Weiss, Units of integral group rings of metabelian groups, J. Algebra 73 (1981), 167-185.

3. E. Jespers and G. Leal, Describing units of integral group rings of some 2-groups, Comm. Algebra 19 (1991), 1809-1827.

4. E. Jespers, G. Leal and M. M. Parmenter, Bicyclic and Bass cyclic units in group rings, Canad. Math. Bull. to appear.

5. M. Newman, Integral matrices (Academic Press, 1972).

6. M. M. Parmenter, Torsion-free normal complements in unit groups of integral group rings, C.R. Math. Rep. Acad. Sci. Canada 12 (1990), 113-118.

7. H. Pollard and H. G. Diamond, The theory of algebraic numbers, The Carus Mathematical Monographs (Mathematical Association of America, 1975).

8. J. Ritter and S. K. Sehgal, Generators of subgroups of $U(\mathbf{Z G )}$, Representation theory, group rings, and coding theory, Contemp. Math. 93 (Amer. Math. Soc., 1989), 331-347.

9. J. Ritter and S. K. Sehgal, Construction of units in integral group rings of finite nilpotent groups, Trans. Amer. Math. Soc. 324 (1991), 603-621.

10. S. K. Sehgal, Topics in group rings (Marcel Dekker, 1978).

11. S. K. Sehgal, Units of integral group rings-A survey, preprint.

Department of Mathematics and Statistics

MEMORIAL UNIVERSITY OF NEWFOUNDLAND

St. JOHN'S, NEWFOUNDLAND

CANADA A1C 5S7 